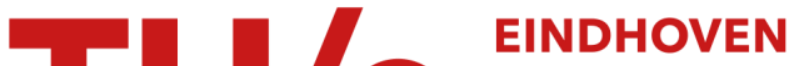 \\ UNIVERSITY OF \\ TECHNOLOGY
}

\section{Two-dimensional estimation of the electrohysterographic conduction velocity}

Citation for published version (APA):

Rabotti, C., \& Mischi, M. (2010). Two-dimensional estimation of the electrohysterographic conduction velocity. In Proceedings on the 32nd Annual International Conference, Buenos Aires, Argentina, 1-4 September, 2010 (pp. 4262-4265). Institute of Electrical and Electronics Engineers.

Document status and date:

Published: 01/01/2010

\section{Document Version:}

Publisher's PDF, also known as Version of Record (includes final page, issue and volume numbers)

\section{Please check the document version of this publication:}

- A submitted manuscript is the version of the article upon submission and before peer-review. There can be important differences between the submitted version and the official published version of record. People interested in the research are advised to contact the author for the final version of the publication, or visit the $\mathrm{DOI}$ to the publisher's website.

- The final author version and the galley proof are versions of the publication after peer review.

- The final published version features the final layout of the paper including the volume, issue and page numbers.

Link to publication

\section{General rights}

Copyright and moral rights for the publications made accessible in the public portal are retained by the authors and/or other copyright owners and it is a condition of accessing publications that users recognise and abide by the legal requirements associated with these rights.

- Users may download and print one copy of any publication from the public portal for the purpose of private study or research.

- You may not further distribute the material or use it for any profit-making activity or commercial gain

- You may freely distribute the URL identifying the publication in the public portal.

If the publication is distributed under the terms of Article 25fa of the Dutch Copyright Act, indicated by the "Taverne" license above, please follow below link for the End User Agreement:

www.tue.nl/taverne

Take down policy

If you believe that this document breaches copyright please contact us at:

openaccess@tue.nl

providing details and we will investigate your claim. 


\title{
Two-dimensional estimation of the electrohysterographic conduction velocity
}

\author{
Chiara Rabotti and Massimo Mischi
}

\begin{abstract}
Propagation of action potentials (APs) through an adequate number of uterine muscle cells induces contraction of the uterus. Monitoring uterine contractions, as the first sign of labor, can provide important information on the course of pregnancy and delivery. Unfortunately, current monitoring methods are affected by serious limitations. The electrohysterogram (EHG), which is the noninvasive recording of the APs propagating through the uterine smooth muscle cells, is here analyzed as a potential alternative to current methods. We focus on estimating the conduction velocity (CV) of surface APs extracted from an EHG recorded in a multielectrode configuration. In this work, a two-dimensional, 64-channel, high density electrode grid is used. Maximum likelihood methods are employed for analyzing the EHG AP propagation in two dimensions. The use of different weighting strategies of the derived cost function is introduced to deal with poor interchannel signal similarity. The presented methods were evaluated by specific simulations proving the best weighting strategy to lead to an accuracy improvement of 58\%. EHG measurements on women with uterine contractions confirmed the feasibility of the method by leading to values of conduction velocity within the expected physiological range.
\end{abstract}

\section{INTRODUCTION}

During pregnancy and delivery accurate monitoring of the uterine contractions can allow timely intervention when risks, e.g., a preterm labor threat, are detected and can improve the effectiveness of the required treatment. However, current methods impose a compromise between reliability and noninvasiveness and alternative techniques are needed [1].

The electrohysterogram (EHG) is the noninvasive measurement of the electrical activity that, by propagating from cell to cell in the form of action potentials (APs), initiates uterine contractions. Previous literature demonstrated that the EHG has great potential for monitoring labor, predicting the delivery time, and support timely treatment of preterm labor. In particular, as AP propagation is among the root causes of a uterine contraction, some parameters derived by the EHG analysis such as conduction velocity (CV), can be predictive of preterm labor.

The uterine muscle, the myometrium, is composed of smooth muscle cells. Differently from skeletal muscles, that present an anatomical direction of AP propagation parallel to the fiber orientation, the direction of propagation of the EHG individual APs is a priori unknown [2]. APs usually occur in bursts. Each burst correspond to a single mechanical contraction of the uterus. Most of the previous literature focused on the analysis of the entire burst and only few

Chiara Rabotti and Massimo Mischi are with the Department of Electrical Engineering, Eindhoven University of technology, the Netherlands C.Rabotti@tue.nl studies were dedicated to the analysis of single surface APs [3], [4], [5], i.e., single spikes extracted from the EHG. However, in-vitro studies have demonstrated that individual surface APs propagate for longer distance and with higher conduction velocity $(\mathrm{CV})$ at parturition than at preterm [3]. Furthermore, as different APs have different propagation properties even within the same burst, analysis of single AP $\mathrm{CV}$ can lead to more extensive information compared to the whole burst analysis.

In this paper we focus on a method for estimating the CV of single APs extracted from EHGs. The EHG is recorded by a high-density electrode grid that integrates a larger number of electrodes (64) with a reduced surface and smaller interelectrode distance with respect to previous studies. Due to the a priori unknown AP direction of propagation, the bidimensional arrangement of the electrodes on the grid $(8 \times 8)$ permits to estimate any of the possible $\mathrm{CV}$ directions along the plane parallel to the abdominal surface.

The standard methods, available from electromyography, for the measurement of the AP CV can be divided in four major categories [6]: cross-correlation function maximization [7], phase difference (PD) [8], maximum likelihood (ML) [9], and the detection of spectral dips [10]. Due to the $a$ priori unknown direction of propagation, implementation of these methods for EHG CV analysis requires their extension to two dimensions. Furthermore, given the low frequency content of EHG signals (usually below $1 \mathrm{~Hz}$ ) and the large number of channels to be processed, implementing the algorithms in the frequency domain allows using lower sampling frequency while obtaining $\mathrm{CV}$ estimation without any resolution limit.

Besides cross-correlation function maximization, the aforementioned methods are implemented in the frequency domain. Among them, the application of a multidip approach to our measurements is complicated by the varying direction of propagation of the uterine APs and by the necessity of extending the method to two dimensions. We have eventually chosen the ML method as it permits, differently from the PD method, a complete exploitation of our multichannel measurements and favors an increased robustness to poor SNR [9]. The ML estimation is equivalent to a mean square error minimization. We propose an improved version of the ML method by weighting the derived error. A set of weights is automatically determined based on SNR estimates at each channel. Two different weighting approaches are here presented and compared. Furthermore, the methods are extended to two dimensions, permitting to estimate amplitude and direction of the CV. The proposed methods were validated 


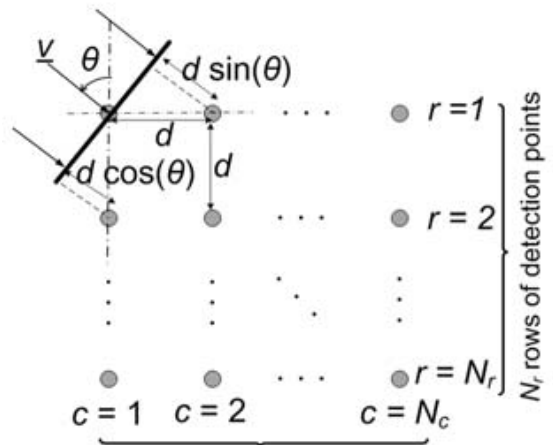

$N_{c}$ columns of detection points

Fig. 1. Schematic description of the model.

on simulated and real signals recorded from women in labor.

\section{METHODOLOGY}

\section{A. Maximum likelihood method}

Following the schematic representation of Fig. 1, we assume the EHG to propagate with velocity $v$ and with incidence angle $\theta(\theta \in[-\pi, \pi])$ with respect to the vertical axis of the electrode grid. Due to the size of the electrode grid, which is of the order of the signal wavelength [5], we can assume the EHG AP to be a planar wave. The signal is detected by $N_{r}$ rows and $N_{c}$ columns of electrodes. Assuming that the same signal shape $s(n)$ is measured at each channel, the adopted ML method is developed under the hypothesis that the signal $x_{r c}$ measured at the channel $(r, c)$ in the $r^{t h}$ row $\left(r \in\left[1,2, \ldots, N_{r}\right]\right)$ and $c^{\text {th }}$ column $\left(c \in\left[1,2, \ldots, N_{c}\right]\right)$ of the electrode grid can be modeled as

$$
x_{r c}(n)=s\left(n-(r-1) \tau_{r}-(c-1) \tau_{c}\right)+w_{r c}(n),
$$

where $n$ indicates the time sample $(n \in[1,2, \ldots, N])$ and $w_{r c}(n)$ is white Gaussian noise with variance $\sigma_{r c}^{2}$ that is present at channel $(r, c)$. The choice of the noise model is supported by the narrow band nature of the signal of interest. As from (1), in each channel $(r, c)$ the reference signal shape $s(n)$ is delayed by $\tau_{r}$ and $\tau_{c}$ time samples relative to the previous row and column, respectively.

The $\mathrm{CV}$ calculation requires the estimation of $\left(\tau_{r}, \tau_{c}\right)$, which can be obtained by the maximization of the probability density function $p\left(\left(\tau_{r}, \tau_{c}\right) \mid x_{r c}(n), s(n)\right)$. Using Bayesian inference and assuming $p\left(\tau_{r}, \tau_{c}\right)$ uniform, the maximization of $p\left(\left(\tau_{r}, \tau_{c}\right) \mid x_{r c}(n), s(n)\right)$ corresponds to the maximization of the probability $p\left(x_{r c}(n) \mid\left(\tau_{r}, \tau_{c}\right), s(n)\right)$ of the samples of the signal $x_{r c}(n)$, given the row and column sample delays, $\tau_{r}$ and $\tau_{c}$, and the reference shape $s(n)$ [11].

Since the signals $x_{r c}(n)$ are only available for discrete values of $\tau_{r}$ and $\tau_{c}$, maximization of $p\left(x_{r c}(n) \mid\left(\tau_{r}, \tau_{c}\right), s(n)\right)$ results in a discrete estimate of the optimum $\left(\tau_{r}, \tau_{c}\right)$, which depends on the sampling rate. By using Parseval's equality, $p\left(x_{r c}(n) \mid\left(\tau_{r}, \tau_{c}\right), s(n)\right)$ can be transformed in the frequency domain, where $\tau_{r}$ and $\tau_{c}$ become continuous phase coefficient [6]. Indicating by $X_{r c}(f)$ and $S(f)$ the Fourier transform of the signal recorded at the channel $(r, c)$ and of the reference shape, respectively, maximization of $p\left(x_{r c}(n) \mid\left(\tau_{r}, \tau_{c}\right), s(n)\right)$ corresponds to minimization of the cost function $E^{2}\left(\tau_{r}, \tau_{c}\right)$, with

$$
\begin{array}{r}
E^{2}\left(\tau_{r}, \tau_{c}\right)=\frac{2}{N} \sum_{r=1}^{N_{r}} \sum_{c=1}^{N_{c}} \sum_{f=1}^{N / 2}\left[X_{r c}(f)+\right. \\
\left.-S(f) e^{-j 2 \pi f\left[(r-1) \tau_{r}+(c-1) \tau_{c}\right]}\right]^{2} .
\end{array}
$$

The shape function $S(f)$ can be estimated as the average of all the channels $X_{r c}(f)$ after alignment, i.e.,

$$
\widehat{S}(f)=\frac{1}{N_{c} N_{r}} \sum_{r=1}^{N_{r}} \sum_{c=1}^{N_{c}} X_{r c}(f) e^{j 2 \pi f\left[(r-1) \tau_{r}+(c-1) \tau_{c}\right]} .
$$

The resulting estimated cost function $\widehat{E}^{2}\left(\tau_{r}, \tau_{c}\right)$ is then

$$
\begin{array}{r}
\widehat{E}^{2}\left(\tau_{r}, \tau_{c}\right)=\frac{2}{N} \sum_{r=1}^{N_{r}} \sum_{c=1}^{N_{c}} \sum_{f=1}^{N / 2}\left[X_{r c}(f)+\right. \\
\left.-\frac{1}{N_{r} N_{c}} \sum_{m=1}^{N_{r}} \sum_{p=1}^{N_{c}} X_{m p}(f) e^{j 2 \pi f\left[(m-r) \tau_{r}+(p-c) \tau_{c}\right]}\right]^{2} .
\end{array}
$$

\section{B. Channel weighting}

The model in (1) is based on the assumption that the signals recorded at different channels are delayed versions of the same reference shape $s(n)$. This assumption, already weak for skeletal muscles [9], is even weaker for the myometrium, where differences in the volume conductor and cell-to-cell conduction path underneath the electrodes may cause shape variations of the propagating APs [2]. In (1) such shape variations are accounted for by the noise term $w_{r c}(n)$. In order to increase the robustness of the $\mathrm{CV}$ estimation to AP shape variations due to the presence of noise, the method is improved by introducing proper weights, $a_{r c} \in \mathbb{R}^{+}$, in the cost function. The resulting weighted cost function $\widehat{E}_{a}^{2}\left(\tau_{r}, \tau_{c}\right)$ is defined as

$$
\begin{aligned}
\widehat{E}_{a}^{2}\left(\tau_{r}, \tau_{c}\right) & =\frac{2}{N} \sum_{r=1}^{N_{r}} \sum_{c=1}^{N_{c}} \sum_{f=1}^{N / 2}\left[a _ { r c } \left(X_{r c}(f)+\right.\right. \\
& \left.\left.-\widehat{S}(f) e^{-j 2 \pi f\left[(r-1) \tau_{r}+(c-1) \tau_{c}\right]}\right)\right]^{2} .
\end{aligned}
$$

The weights are chosen to be inversely proportional to the standard deviation of the channel noise $\sigma_{r c}$ [12], i.e,

$$
a_{r c}=\frac{A}{\sigma_{r c}}=\frac{A}{\frac{2}{N} \sqrt{\sum_{f=1}^{N / 2}\left|W_{r c}(f)\right|^{2}}},
$$

were $A$ indicates a proper scaling factor to normalize the weight sum to 1 . For the expression of $a_{r c}$ in the frequency domain (last term of (6)) Parseval's equality is used, where $\left|W_{r c}(f)\right|^{2}$ is the noise power spectrum in the considered channel $(r, c)$.

In order to obtain an estimate of the noise power for the generic channel $(r, c)$, the model in (1) is expressed in the temporal frequency domain $f$ as

$$
X_{r c}(f)=S(f) e^{-j 2 \pi f\left[(r-1) \tau_{r}+(c-1) \tau_{c}\right]}+W_{r c}(f) .
$$


By assuming the reference shape $S(f)$ and the noise $W_{r c}(f)$ to be uncorrelated, the noise can be estimated from

$$
\sum_{f=1}^{N / 2} X_{r c}(f) \cdot X_{r c}^{*}(f)=\sum_{f=1}^{N / 2} S(f) \cdot S^{*}(f)+\sum_{f=1}^{N / 2}\left|W_{r c}(f)\right|^{2},
$$

where $(\cdot)^{*}$ is the conjugate operator. The noise power derived by (8) can then be substituted in (6) to provide the weights

$$
a_{r c}=\frac{A}{\frac{2}{N} \sqrt{\sum_{f=1}^{N / 2}\left(X_{r c}(f) \cdot X_{r c}^{*}(f)-S(f) \cdot S^{*}(f)\right)}} .
$$

The shape $\widehat{S}(f)$ defined in (3) as the average of the aligned signals $X_{c r}$, which is used as estimate of the reference signal $\widehat{S}(f)$ in (5), can be employed in (9).

Alternatively, the estimate $\widehat{S}_{w}(f)$ of the reference shape $S(f)$ in (9) can be calculated as the weighted average of the signals $X_{r c}(f)$, i.e,

$$
\widehat{S}_{w}(f)=\sum_{r=1}^{N_{r}} \sum_{c=1}^{N_{c}} a_{r c}^{w} \cdot X_{r c}(f) e^{j 2 \pi f\left[(r-1) \tau_{r}+(c-1) \tau_{c}\right]} .
$$

Using $\widehat{S}_{w}(f)$ as estimate of $S(f)$ in (9), the alternative channel weights $a_{r c}^{w}$ are defined as

$$
a_{r c}^{w}=\frac{A}{\frac{2}{N} \sqrt{\sum_{f=1}^{N / 2} X_{r c}(f) \cdot X_{r c}^{*}(f)-\widehat{S}_{w}(f) \widehat{S}_{w}^{*}(f)}},
$$

and using (10) for $\widehat{S}_{w}(f)$ and $\widehat{S}_{w}^{*}(f),(11)$ can be expressed as

$$
a_{r c}^{w}=\frac{A}{\frac{2}{N} \sqrt{\sum_{f=1}^{N / 2} X_{r c}(f) \cdot X_{r c}^{*}(f)-\sum_{r=1}^{N_{r}} \sum_{c=1}^{N_{c}}\left(a_{r c}^{w}\right)^{2} \cdot X_{r c}(f) X_{r c}^{*}(f)}} .
$$

The $N_{r} \cdot N_{c}$ equations of the same form as (12), which holds for each channel $(r, c)$, and the condition on the weight sum

$$
\sum_{r=1}^{N_{r}} \sum_{c=1}^{N_{c}} a_{r c}^{w}=1
$$

lead to a system of $N_{r} \cdot N_{c}+1$ linearly independent equations, where the $N_{r} \cdot N_{c}$ unknown weights and the scaling factor $A$ can be univocally derived. Using the same weights $a_{r c}^{w}$ for the cost function and the reference shape in (9) leads to the following expression of the estimated alternative cost function $\widehat{E}_{a^{w}}^{2}\left(\tau_{r}, \tau_{c}\right)$,

$$
\begin{array}{r}
\widehat{E}_{a^{w}}^{2}\left(\tau_{r}, \tau_{c}\right)=\frac{2}{N} \sum_{r=1}^{N_{r}} \sum_{c=1}^{N_{c}} \sum_{f=1}^{N / 2}\left[a _ { r c } ^ { w } \left(X_{r c}(f)+\right.\right. \\
\left.\left.-\widehat{S}_{w}(f) e^{-j 2 \pi f\left[(r-1) \tau_{r}+(c-1) \tau_{c}\right]}\right)\right]^{2},
\end{array}
$$

where, differently from the cost function $E^{2}\left(\tau_{r}, \tau_{c}\right)$ in (5), the weights $a_{r c}^{w}$ are calculated using the weighted average $\widehat{S}_{w}(f)$ of the signals $X_{r c}(f)$ as estimate of the reference shape $S(f)$.
TABLE I

DELAY ESTIMATES FOR DIFFERENT ANGLES OF INCIDENCE.

\begin{tabular}{|l|ll|ll|ll|ll|}
\hline \multirow{2}{*}{ Cost function } & \multicolumn{2}{|c|}{$\theta=0$} & \multicolumn{2}{c|}{$\theta=\pi / 12$} & \multicolumn{2}{c|}{$\theta=\pi / 6$} & \multicolumn{2}{c|}{$\theta=\pi / 4$} \\
& $\mathrm{SD}_{\mathrm{r}}$ & $\mathrm{SD}_{\mathrm{c}}$ & $\mathrm{SD}_{\mathrm{r}}$ & $\mathrm{SD}_{\mathrm{c}}$ & $\mathrm{SD}_{\mathrm{r}}$ & $\mathrm{SD}_{\mathrm{c}}$ & $\mathrm{SD}_{\mathrm{r}}$ & $\mathrm{SD}_{\mathrm{c}}$ \\
\hline$\widehat{E}^{2}\left(\tau_{r}, \tau_{c}\right)$ & $7.0 \mathrm{~ms}$ & $5.5 \mathrm{~ms}$ & $6.2 \mathrm{~ms}$ & $5.4 \mathrm{~ms}$ & $6.2 \mathrm{~ms}$ & $5.9 \mathrm{~ms}$ & $6.4 \mathrm{~ms}$ & $5.6 \mathrm{~ms}$ \\
$\widehat{E}_{a}^{2}\left(\tau_{r}, \tau_{c}\right)$ & $3.2 \mathrm{~ms}$ & $2.5 \mathrm{~ms}$ & $3.0 \mathrm{~ms}$ & $2.8 \mathrm{~ms}$ & $2.9 \mathrm{~ms}$ & $3.0 \mathrm{~ms}$ & $3.0 \mathrm{~ms}$ & $3.1 \mathrm{~ms}$ \\
$\widehat{E}_{a^{w}}^{2}\left(\tau_{r}, \tau_{c}\right)$ & $2.7 \mathrm{~ms}$ & $2.3 \mathrm{~ms}$ & $2.5 \mathrm{~ms}$ & $2.4 \mathrm{~ms}$ & $2.5 \mathrm{~ms}$ & $2.5 \mathrm{~ms}$ & $2.7 \mathrm{~ms}$ & $2.6 \mathrm{~ms}$ \\
\hline
\end{tabular}

For validation, the three different cost functions $E^{2}\left(\tau_{r}, \tau_{c}\right)$, $E_{a}^{2}\left(\tau_{r}, \tau_{c}\right)$, and $\widehat{E}_{a^{w}}^{2}\left(\tau_{r}, \tau_{c}\right)$ were compared on simulated and real signals. For the minimization of the cost functions, the Nelder-Mead Simplex search method was used [13].

\section{RESULTS}

\section{A. Simulated signals}

The presented CV-estimation methods are evaluated by means of simulations based on real signals. A time interval of $10 \mathrm{~s}$ including a complete EHG surface AP was extracted from a real EHG recording to obtain the reference shape $s(n)$. This signal was then artificially delayed to simulate the measurement of the same AP by the other electrodes on the grid. A velocity of $4 \mathrm{~cm} / \mathrm{s}$ and four different angles of incidence, equal to $0, \pi / 12, \pi / 6$, and $\pi / 4$, were considered.

For each simulated angle of incidence, 1000 different white Gaussian noise sequences were generated and added to each channel; the SNR was randomly distributed among the channels according to a Gaussian probability density function with the same mean and standard deviation, equal to $5.88 \mathrm{~dB}$ and $7.41 \mathrm{~dB}$, respectively, as estimated from our real signals by (8).

The CV-estimates were calculated by the ML method alone, and after the use of the two different weighting strategies. The standard deviations of the error for the row delay $\tau_{r}\left(\mathrm{SD}_{\mathrm{r}}\right)$ and the column delay $\tau_{c}\left(\mathrm{SD}_{\mathrm{c}}\right)$ are reported in Table I for each simulated angle of incidence and for the different used cost functions. The errors were all unbiased. On average, weighting the cost function reduced the standard deviation of the error by $51.06 \% \pm 3.26 \%$. Weighting of both the cost function and of the reference shape provided an average improvement of $58 \% \pm 2.25 \%$.

\section{B. Real signals}

In order to assess the measurement feasibility, after approval of the medical committee of the hospital, measurements were performed at the Máxima Medical Center in Veldhoven (the Netherlands) on five women who signed an informed written consent. The EHG signal was recorded and digitized at $1024 \mathrm{~Hz}$ by a Refa system (TMS International, Enschede, the Netherlands) comprising a multichannel amplifier for electrophysiological signals and a grid of 64 (8x8) high-density (HD) electrodes $(1 \mathrm{~mm}$ diameter, $4 \mathrm{~mm}$ inter-electrode distance). A more detailed description of the measurement setup can be found in [5].

Given the narrow-band nature of the EHG signal, the acquired signals were band-pass filtered between 0.1 and $0.8 \mathrm{~Hz}$, respectively [5]. This permitted to suppress most 


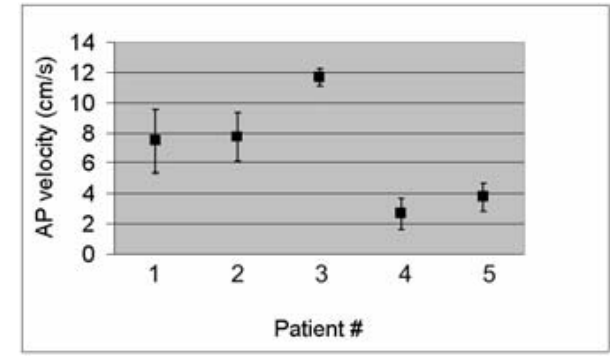

Fig. 2. Mean and standard deviation of the $\mathrm{CV}$ amplitude for all patients.

of the noise introduced by respiration, maternal electrocardiogram, and abdominal electromyogram. The filtered signals could therefore be downsampled from 1024 to $16 \mathrm{~Hz}$ without introducing aliasing and reducing significantly the computational complexity of the following analysis. During contractions, time segments were visually inspected on the preprocessed signals and two APs were determined per each woman. The AP visual selection aimed at excluding possible circulating excitations and re-entries [14].

The method comprising the minimization of the cost function $\widehat{E}_{a^{w}}^{2}\left(\tau_{r}, \tau_{c}\right)$ was applied on the entire $8 \times 8$ electrode matrix. The average and standard deviation of the velocity amplitude are reported in Fig. 2 for all patients. On average, we found vertical and horizontal components of the velocity amplitude equal to $3.6 \pm 3.4 \mathrm{~cm} / \mathrm{s}$ and $5 \pm 3.6 \mathrm{~cm} / \mathrm{s}$, respectively. These estimates are within the expected physiological range [2]. Concerning the wave incidence angle, as it was previously demonstrated [4], a preferred direction of propagation of single APs could not be highlighted and, even within the same contraction, different incidence angles were detected for different APs.

\section{DISCUSSION AND CONCLUSION}

The measurement of the EHG surface AP CV is here proposed. The use of an electrode matrix permits estimating the $\mathrm{CV}$ vector in two dimensions. This is an important aspect in EHG measurements because, differently from electromyographic $\mathrm{CV}$ measurements, the EHG $\mathrm{CV}$ direction is not known a priori. For the signal analysis we propose a ML method, which is implemented in two dimensions and comprises the use of weights in the cost function. The weight values depend on the estimated SNR.

Results on simulated signals show that the estimate accuracy is significantly improved by the use of weights. Among the two different weighting strategies that were proposed, the use of the same weights for estimating the reference signal shape and for the cost function results in more accurate estimates. As compared to the ML method alone, on average, the error variance diminished by $58 \%$.

The method feasibility was preliminarily confirmed by measurements on five women at term with uterine contractions. Calculation of the $\mathrm{CV}$ amplitude led to values that are within the expected physiological range [2], [3], [4]. As for the incidence angle of the surface APs, differently from what is reported for the propagation of the whole electrical burst [15], we could not highlight a most frequent direction of the AP propagation pattern even within the same contraction. The same variability in both origin and direction of the AP propagation pattern has been previously observed in in-vivo and in-vitro studies on the uterus, and, at least during labor [4], it seems physiological.

In conclusion, our results show that the proposed ML method is suitable for the two dimensional estimation of the electrohysterographic AP conduction velocity. Moreover, the use of weights for both the reference shape and the cost function leads to more accurate estimates than the use of the ML alone and should therefore be preferred. In general, the proposed methodologies are suitable also for the analysis of other signals, and are particularly advantageous in the presence of poor SNRs and a priori unknown propagation pattern. In the context of EHG signal analysis, the method opens new possibilities for future clinical studies aimed at assessing the CV-vector dynamics and its value for analysis of the pregnancy course and, most importantly, for prediction of preterm delivery.

\section{ACKNOWLEDGMENTS}

For the signal acquisition, the authors gratefully acknowledge the contribution of the department of Obstetric and Gynecology of the Máxima Medical Center (Veldhoven, NL) and TMS International (Enschede, NL).

\section{REFERENCES}

[1] R. Garfield et al., "Methods and devices for the management of term and preterm labor," Ann. NY Acad.Sci., vol. 943, pp. 203-224, 2001.

[2] D. Devedeux et al., "Uterine electromyography: a critical review," Am. J. Obstet. Gynecol., vol. 169, pp. 1636-1653, 1993.

[3] S. Miller et al., "Improved propagation in myometrium associated with gap junctions during parturition," Am. J. Physiol. Cell. Physiol., vol. 256, pp. C130-C141, 1989.

[4] W. Lammers et al. "Patterns of electrical propagation in the intact guinea pig uterus," Am. J. Physiol. Regul. Integr. Comp. Physiol., vol. 294, pp. R919-R928, 2008.

[5] C. Rabotti et al., "Modeling and identification of the electrohysterographic volume conductor by high-density electrodes," IEEE Trans. Biomed. Eng., vol. 57, pp. 519-527, 2010.

[6] D. Farina and R. Merletti, "Methods for estimating muscle fibre conduction velocity from surface electromyographic signals," Med. Biol. Eng. Comput., vol. 42, pp. 432-445, 2004.

[7] P. Parker and R. Scott, "Statistics of the myoelectric signal from monopolar and bipolar electrodes," Med. Biol. Eng., vol. 11, pp. 591596, 1973.

[8] I. Hunter et al., "Estimation of the conduction velocity of muscle action potentials using phase and impulse response function techniques," Med. Biol. Eng., vol. 25, pp. 121-126, 1987.

[9] D. Farina et al. "Estimation of single motor unit conduction velocity from surface electromyogram signals detected with linear electrode arrays," Med. Biol. Eng. Comput., vol. 39, pp. 225-236, 2001.

[10] P. Lynn, "Direct on-line estimation of muscle fiber conduction velocity by surface electromyography," IEEE Trans. Biomed. Eng., vol. 26, pp. 564-571, 1979.

[11] C. Bishop, Pattern Recognition and Machine Learning. Springer, 2006.

[12] D. Manolakis et al., Statistical and Adaptive Signal Processing. Norwood, MA: Artech house, 2005.

[13] J. Lagarias et al., "Convergence properties of the Nelder-Mead simplex method in low dimensions," SIAM J. Optim., vol. 9, pp. 112-147, 1998.

[14] W. Lammers, "Circulating excitations and re-entry in the pregnant uterus." Eur. J. Physiol., vol. 433, pp. 287-293, 1997.

[15] C. Rabotti et al., "Inter-electrode delay estimators for electrohysterographic propagation analysis," Physiol. Meas., vol. 30, pp. 745-761, 2009. 